\title{
Elaboração de fluxograma assistencial para avaliação de dor em crianças e adolescentes
}

\section{Care flowchart elaboration for pain assessment in children and adolescents}

\author{
DOI: $10.46919 / \operatorname{archv1n6-013}$
}

Recebimento dos originais: 01/09/2020

Aceitação para publicação: 30/10/2020

\section{Anndreya Marques de Souza Rodrigues}

Graduada em Enfermagem pela Escola Superior em Ciências da Saúde Instituição: Escola Superior de Ciências da Saúde.

Endereço: Edifício Fepecs. SMHN Conjunto A Bloco 01 - Asa Norte, Brasilia - DF, Brasil. CEP: 70710100

Email: anndreyamarques@gmail.com.

\section{Amanda Costa Melo}

Especialista em Enfermagem do Trabalho pela Escola Bahiana de Medicina e Saúde Pública Instituição: Secretaria de Saúde do Estado da Bahia

Endereço: $4^{\mathrm{a}}$ Avenida Centro Administrativo da Bahia, 400 - Centro Administrativo da Bahia, Salvador BA, 40301-110

E-mail: preceptoraamanda@gmail.com

\section{Ruth Geralda Germana Martins}

Mestre em Ciências da Tecnologia pela Universidade de Brasília

Instituição: Secretaria de Saúde do Distrito Federal

Endereço: STN - Asa Norte, Brasília - DF, Brasil. CEP: 70086-900

E-mail: ruth.germana@gmail.com

\section{Manuela Costa Melo}

Doutora em Enfermagem pela Universidade de Brasília Instituição: Escola Superior de Ciências da Saúde

Edifício Fepecs. SMHN Conjunto A Bloco 01 - Asa Norte, Brasilia - DF, Brasil. CEP: 70710-100

E-mail: melomanuela91@ gmail.com

\section{Ana Socorro de Moura}

Mestre em Ciências da Saúde Universidade de Brasília Instituição: Escola técnica na Brasília

Endereço: Edifício Fepecs. SMHN Conjunto A Bloco 01 - Asa Norte, Brasilia - DF, Brasil. CEP: 70710100

E-mail: prof.ana10@gmail.com

\section{Lara Mabelle Milfont Boeckmann}

Doutora em Enfermagem pela Universidade de Brasília

Instituição: Universidade de Brasília,

Endereço: Campus Universitário Darcy Ribeiro - Asa Norte, Brasília-DF, Brasil. CEP: 70910-900

E-mail: laramilfont@gmail.com 


\title{
RESUMO
}

Objetivo: Elaborar fluxograma assistencial que possibilite a avaliação, mensuração e tratamento não farmacológico para alivio da dor em crianças e adolescentes. Método: Realizado Pesquisa Aplicada, por meio de revisão bibliográfica e utilização da matriz estabelecida pela Secretaria de Saúde do Distrito Federal para confecção de fluxogramas assitenciais e clínicos. Estudo realizado entre junho de 2018 a junho de 2019, utilizou-se as escalas FLACC, Wong-Backer Faces e Escala Visual Analógica. Resultados: Por meio da matriz estabelecida foi desenvolvido o fluxograma assistencial com identificação do fluxograma para avaliação de acordo com a faixa etária, com a identificação de medidas não farmacológicas. Conclusão: Apresentação do fluxograma propõe facilidade na assistência de profissionais na sua prática clínica. Faz-se necessário que os profissionais entendam a dor como um evento complexo, que demanda educação permanente, para uma intervenção precoce e humanizada, garantindo a eficiência do cuidado.

Palavra-chave: Cuidados de enfermagem, Dor, Saúde da Criança, Manejo da dor.

\begin{abstract}
Objective: To develop a care flowchart that allows the evaluation, measurement and non-pharmacological treatment for pain relief in children and adolescents. Method: Applied Research was carried out through a literature review and use of the matrix established by the Health Department of the Federal District to make assistential and clinical flowcharts. A study conducted between June 2018 and June 2019 used the FLACC, Wong-Backer Faces and Visual Analog Scales. Results: Through the established matrix, the care flowchart was developed with identification of the flowchart for evaluation according to age group, with the identification of non-pharmacological measures. Conclusion: Presentation of the flowchart proposes ease in the care of professionals in their clinical practice. It is necessary that professionals understand pain as a complex event, which demands permanent education, for an early and humanized intervention, ensuring efficiency,
\end{abstract}

Keywords: Nursing Care, Pain, Child Health, Pain Management.

\section{INTRODUÇÃO}

O processo de internação infantil é um fato que marca a criança e o adolescente, uma vez que, nesse momento elas se sentem vulneráveis, por estarem afastadas de seu ambiente familiar o que gera mudanças em sua rotina diária. Entre as experiências desagradáveis vivenciadas durante o atendimento em serviços de saúde ou internação hospitalar está a dor, que por vezes é vivenciada a partir de procedimentos realizados ou pelo próprio processo patológico ${ }^{(1-3)}$.

O fenômeno doloroso é definido pela International Association for the Study of Pain (IASP) “Associação Internacional para o Estudo da Dor”, como "uma sensação ou experiência emocional desagradável, associada a um dano real ou potencial descrito em termos de tal dano" (4). Em 2011, essa mesma definição foi revisada e acrescentada que "a inabilidade de comunicar verbalmente a dor não nega a possibilidade de o indivíduo a ter experimentado e a sua necessidade de receber tratamento adequado para seu alívio"(4-6).

Diante de tal fato, a dor foi incluída como $5^{\circ}$ sinal vital e de extrema importância, uma vez que durante a prática clínica, aplicada a unidades de internação pediátrica, sua avaliação e mensuração se 
tornam complexas devido a qualidade da caracterização da dor, pela falta ou dificuldade de verbalização desta por parte do paciente, além da subjetividade. Devido a isso os profissionais precisam de parametros confiáveis para mensuração da dor de acordo com a faixa etária ${ }^{(3,7-10)}$.

Dessa maneira, é de imprescindível importância a elaboração de fluxograma de saúde que possibilite aos profissionais realizarem a condução do evento doloroso por meio de escalas validadas, para que assim, possam sistematizar suas ações no cuidado à criança e ao adolescente hospitalizado ${ }^{(11-12)}$. Entre várias escalas validadas existentes, podemos citar as Escalas Face, Legs, Activity, Cry, Consolablity (FLACC), Wong-Backer faces e Escala visual Analógica (EVA), que podem ser utilizadas, cada uma dentro de sua faixa etária, pois cada uma dessas possuem características peculiares para sua aplicação prática $^{(13-15)}$.

$\mathrm{O}$ cuidado à criança e ao adolescente dirige-se para a organização da assistência, portanto este estudo justifica-se por apresentar um fluxograma assistencial no intuito de instrumentalizar de forma sistemática à equipe de saúde e assim, contribuir para o aprimoramento do grupo sobre a temática e melhoria da qualidade da assistência, excelência e humanização do cuidado. Para proporcionar um tratamento individualizado adequado e resolutivo a fim de minimizar o desconforto e o efeito doloroso a essas crianças iinternadas.

Sendo assim, elaborou-se a seguinte questão norteadora: quais os critérios devem conter em um fluxograma assistencial para alívio da dor em crianças e adolescentes? Diante deste questionamento, o presente estudo teve por objetivo elaborar fluxograma assistencial que possibilite a avaliação, mensuração e tratamento não farmacológico para alívio da dor em crianças e adolescentes.

\section{MATERIAL E MÉTODO}

Trata-se de um estudo na abordagem de Pesquisa Aplicada para elaboração de fluxograma assistencial em enfermagem ${ }^{(16)}$. Com relação a abrangência, foi elaborado para ser aplicado em crianças e adolescentes internados na unidade de pediatria, tanto no pronto-socorro, como em unidade de internação.

$\mathrm{Na}$ sequência, o referencial teórico que norteou a elaboração do fluxograma assistencial foi a matriz direcionada pelas normas estabelecidas pela Secretaria de Estado de Saúde do Distrito Federal $(\mathrm{SES}-\mathrm{DF})^{(17)}$. A matriz da SES-DF estabelece os aspectos que devem ser seguidos para elaboração de fluxogramas na rede pública, de maneira geral são apresentados em três estapas.

Na Etapa 1 ocorreu uma ampla revisão da literatura disponível com texto na íntegra e acesso gratuito. Na Etapa 2, ocorreu elaboração do fluxograma com informações do diagnóstico clínico da população alvo, critérios de inclusão e exclusão de participantes, a conduta preventiva com tratamentos não farmacológicos e elaboração do fluxograma assistencial. E a última, a Etapa 3, responsável pela informação dos benefícios esperados; monitorização, regulação/controle/avaliação pelo gestor, essa 
última etapa não foi realizada, aguarda estudos futuros para sua aplicação, pois será realizada validação do fluxograma por meio de profissionais da área clínica.

Outros aspectos, presentes na matriz da SES-DF não se aplicaram a este estudo, devido o carater assistencial não farmacológico são: plano terapêutico, critérios de internação, critérios de alta / transferência, cuidados especiais e código internacional de doenças (CID).

Este estudo foi aprovado pelo Comitê de Ética em Pesquisa da Fundação de Ensino e Pesquisa em Ciências da Saúde - FEPECS/ SES/DF, sob $\mathrm{n}^{\circ}$ de parecer 3.105.632. Os aspectos éticos foram atendidos em conformidade com a Resolução do Conselho Nacional de Saúde 466/12.

\section{RESULTADOS}

\subsection{ETAPA 1 - REVISÃO DA LITERATURA}

No aspecto da revisão da literatura foi elaborado a revisão bibliográfica, a coleta de dados ocorreu no período de junho de 2018 a junho de 2019, nas bases de dados: SCIELO, LILACS, BDENF. Os descritores controlados foram identificados no Banco de Descritores em Ciências da Saúde (DeCS) e utilizados os operadores booleanos "AND" e "OR" para realizar os cruzamentos e sistematizar a coleta da amostra. Os termos utilizados foram: Cuidados de enfermagem, Enfermagem, Protocolos de enfermagem, Dor, Saúde da Criança. Os Critérios de inclusão para seleção dos estudos foram os seguintes: estudos nos idiomas inglês e português publicados em texto integralmente indexado nos bancos de dados mencionados. Desta forma, resultaram 29 artigos.

Da leitura realizada foram escolhidos como referêncial teórico as seguntes escalas de dor: escala Face, Legs, Activity, Cry, Consolablity (FLACC) ${ }^{(13)}$, escala WONG-BAKER FACES ${ }^{(14)}$ e Escala Visual Analógica (EVA) para dor (Visual Analogue Scale - VAS) ${ }^{(15)}$.

Um dos instrumentos escolhidos para avaliar e mensurar a dor em recém-nascidos e pediátricos em fase não verbal ou com dificuldades na fala é a escala $\mathrm{FLACC}^{(13)}$. É uma escala desenvolvida pela Universidade de Michigan e é baseada em parâmetros comportamentais, desenvolvida em 1997 e que apresenta cinco categorias de avaliação, que quando somados os scores totalizar o número 10, são elas: expressão facial, movimento de pernas, atividade, choro e consolabilidade. A caracterização da dor se manifesta da seguinte forma: zero a três (dor leve); quatro a seis (dor moderada) e sete a dez (dor intensa) $)^{(13)}$.

Outra escala escolhida como instrumento para avaliação de dor em pacientes internados em unidade pediátrica é a escala WONG-BAKER FACES ${ }^{(14)}$. É uma escala baseada no auto relato para dor aguda, utilizando faces. A faixa etária contemplada é aquela em que os pacientes já estejam na fase verbal, ou seja, crianças a partir de 3 anos. É composta por seis expressões variadas de dor com escores de 0 a 10, onde 0 ou grande sorrisinho demonstra nenhuma sintomatologia para dor e 10, corresponde ao 
desenho de chorar, corresponde a máxima sintomatologia para dor. São atraentes às crianças e fáceis de usar, pois elas podem simplesmente apontar para o rosto que representa como se sentem ${ }^{(14)}$.

Algumas características contribuem para a aplicabilidade desta escala, dentre elas: baixo custo, fácil aplicabilidade, é atrativo às crianças, pais e equipe de saúde. É aplicada da seguinte forma: o profissional da equipe descreve a dor mostrando para criança o que cada rosto representa, em seguida, este profissional pede a criança que identifique o rosto que se adequa a sua dor, conforme instrumento $\operatorname{apresentado}^{(14)}$.

Por fim a última escala escolhida para compor a avaliação e mensuração da dor, neste instrumento de sistematização, é a Escala Visual Analógica (EVA) para dor (Visual Analogue Scale - VAS) ${ }^{(15)}$. Tratase de um instrumento unidimensional podendo ser utilizada para crianças maiores e adolescentes, associada ou não com a escala anteriormente descrita', Trata-se de uma linha com as extremidades numeradas de 0-10. Em uma extremidade da linha é marcada "nenhuma dor" e na outra "pior dor imaginável". Esta última escala pode ou não ser empregada em associação com a escala FACES.

\subsection{ETAPA 2 - ELABORAÇÃO DO FLUXOGRAMA ASSISTENCIAL}

Para elaboração do fluxograma foram considerados a história clínica com informações dos sinais manifestados por crianças e adolescentes, levando-se em consideração a faixa etária, em fase não verbal, tais como, alteração da mimica facial, choro e alteração dos sinais vitais; em crianças em fase verbal e adolescentes, choro e o autorrelato da dor, exames diagnósticos, por meio das escalas de dor já validadas. Preparado para ser utilizado com crianças menores de 14 anos, 11 meses e 29 dias de idade em internação em unidade pediátrica; e excluídas, crianças e adolescentes internados que não apresentam sinais clínicos de dor. No aspecto, tratamento não farmacológico, poderá ser indicado o brinquedo terapêutico, administração de glicose, amamentação, estimulação da sucção nutritiva e contenção facilitada/ enrolamento. O fluxograma assistencial será aplicado as escalas de acordo com cada faixa etária das crianças e adolescentes (figura 1).

Figura 1- Fluxograma de Atendimento 


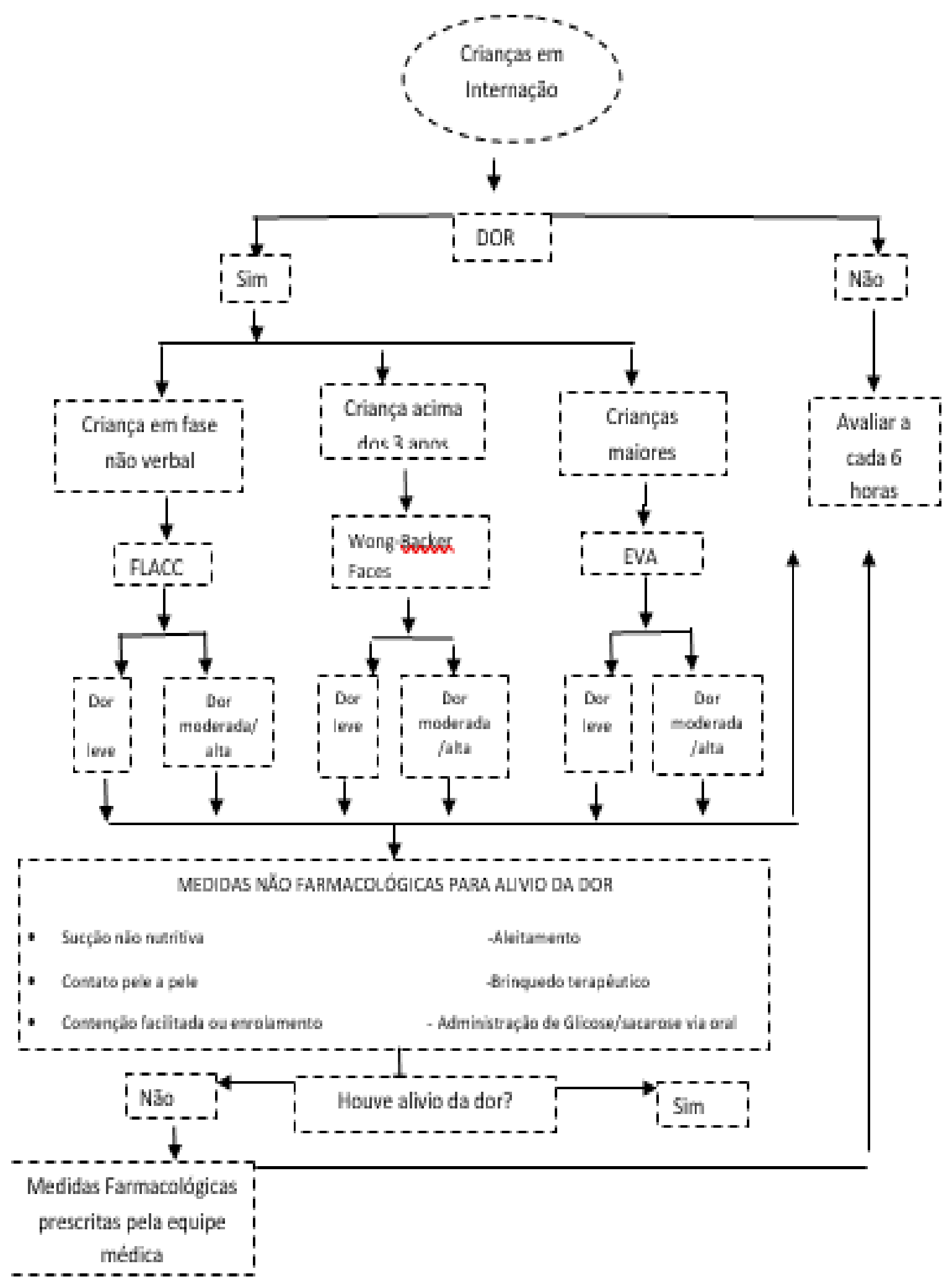

Legenda: FLACC - Face, Legs, Activity, Cry, Consolablity; EVA - Escala Visual Analógica

O fluxograma informa que após a avaliação e mensuração do sinal vital por parte do profissional da saúde, e constatado a presença da dor, métodos não farmacológicos poderão ser empregados no alívio 
dos sintomas. Manejos como: permitir ao acompanhante ficar com a criança/adolescente durante o procedimento, evitar avaliações negativas, oferecer um brinquedo terapêutico a criança / adolescente que permita que seja simulado o procedimento a ser realizado, e assim, o controle da dor pode ser enfatizado neste brinquedo.

Outros manejos como a amamentação, contato pele a pele, estimulação a sucção não nutritiva, contenção facilitada e enrolamento, administração de glicose/sacarose via oral são empregados para o alivio do sintoma. Caso nenhum destes tenha um efeito satisfatório, deverá ser considerada a prescrição de um fármaco por parte da equipe médica para desta forma fornecer o alívio.

\section{DISCUSSÃO}

A inclusão da dor como $5^{\circ}$ sinal vital com a avaliação e o registro apropriado e uma intervenção oportuna e de maneira adequada, por parte dos profissionais de saúde, confere aos pacientes, principalmente os pediátricos, um manejo correto para o controle da dor ${ }^{(7)}$. A avaliação da dor quando acontece no contexto pediátrico, se torna ainda mais complexo e imprescindível, devido à falta e/ou dificuldade de verbalização, além da subjetividade já mencionada ${ }^{(4)}$.

Estudos trazem que os profissionais de saúde, em especial os enfermeiros, encontram uma barreira para a avaliação e manejo da dor, devido ao desconhecimento acerca dos fenômenos dolorosos e mostra que isto provem da falta de conhecimento sobre o assunto e a relevância clínica, o que por sua vez dificulta a implementação de práticas que contribuam para a segurança do paciente. Esse estudo mostra que as avaliações, assim como o seu alívio, necessitam de competências para a promoção de um cuidado efetivo e individualizado. E por ser o enfermeiro, o profissional que está em posição privilegiada, uma vez que está ligado ao cuidado direto do paciente pediátrico, considera-se que este conseguiria avaliar o bem-estar físico e a resposta à terapêutica instituída ${ }^{(3,8-9)}$. Corroborando com este estudo, uma pesquisa mostrou que muitos enfermeiros têm acesso à educação permanente por meio de cursos, contudo, a temática da dor não é incluída na capacitação da equipe de enfermagem ${ }^{(10)}$.

Diante deste fato, é relevante que o enfermeiro saiba identificar, avaliar e tratar a dor, afim de que diminua e/ou evite os eventos dolorosos, contribuindo assim para um restabelecimento mais rápido deste paciente internado. Logo, a elaboração de um fluxograma assistencial, visa a organização das ações do enfermeiro e sua equipe referente a avaliação, manejo e tratamento não farmacológico, possibilitando assim a sistematização da assistência prestada ${ }^{(11)}$.

Desta forma, é recomendada a medição da dor por meio de instrumentos específicos que permitam ao enfermeiro obter escore de avaliação e desta forma planejar o cuidado. E é neste sentido que surgem as escalas de avaliação de dor ${ }^{(12)}$. Para uma avaliação mais fidedigna, o correto é aplicar escalas direcionadas 
para cada faixa etária, pois cada paciente pediátrico tem uma forma diferente de manifestar este sinal vital.

Um estudo desenvolvido com a participação de enfermeiros e pais, traz um comparativo de utilização da escala FLACC, uma vez que estes participantes conseguiriam realizar a avaliação, levandose em conta que o processo é baseado em comportamentos apresentados pelos pacientes, contudo o resultado da pesquisa mostrou que a escala foi melhor aceita e bem utilizada por parte da equipe de enfermagem, uma vez que estes são detentores de competências na área de avaliação ${ }^{(18)}$. Diante deste fato esta escala poderá ser aplicada pela equipe de enfermagem durante a internação hospitalar, utilizando-se do instrumento antes de qualquer procedimento doloroso e após intervenção não farmacológica e farmacológica. O seu preenchimento deve ser realizado a partir da observação atenta do comportamento do paciente em torno de 2 a 3 minutos.

Um estudo clínico aleatório utilizou-se da escala Wong Backer FACES para avaliar o nível de dor antes e após a realização de punção venosa em crianças de 6 a 9 anos. O estudo foi realizado com um grupo controle e um grupo aplicado a intervenção (realidade virtual), e foi constatado que o grupo em que a intervenção foi aplicada obteve um escore entre 1 e 3 dentro da escala aplicada. Neste mesmo estudo evidenciou-se a satisfação dos pais com relação a distração aplicada ${ }^{(19)}$. Outro estudo corrobora com a validade e aplicabilidade desta escala, bem como a diminuição significativa da dor em crianças submetidas a intervenção não farmacológica (doutores da alegria), o que confirma o poder analgésico do riso $^{(20)}$.

Com isso, existe uma equivalência entre a intensidade da dor e a posição que esta foi assinalada na linha, obtendo desta forma uma avaliação numérica ${ }^{(1,21)}$, podendo ou não ser associada a Escala de Faces $^{(15)}$. Estudo realizado após a aplicação de anestésico tópico em 1.070 crianças antes de punção venosa pelos enfermeiros evidenciou diminuição da dor. Neste mesmo estudo observou-se que a partir de um ensaio clínico randomizado controlado com 80 crianças verificou-se estatisticamente a redução da dor após aplicada a EVA, demonstrando desta forma maior satisfação e adequação do processo doloroso por parte da criança ${ }^{(18)}$.

Após a avaliação e mensuração da dor por parte da equipe de enfermagem, esta terá subsídios suficientes para implementar ou não o tratamento não farmacológico. As intervenções não farmacológicas são ações de conforto com a finalidade de prevenir e aliviar o processo doloroso, minimizando desta forma possíveis repercussões negativas ${ }^{(22)}$. Este estudo, traz alguns métodos mais utilizados: amamentação, administração de sacarose/glucose via oral, Sucção não nutritiva, e Técnica de distração.

A amamentação é relevante no que diz respeito ao metodo não farmacológica para alívio de quadro álgico. Um Estudo aponta que um dos componentes do leite materno, o triptofano, um precursor da melatonina, possibilita aumento da endorfina. O odor que melhora a resposta ao choro e a sucção que 
se torna mais efetiva, além do contato, mostrando desta forma que existe relação entre estes fatores com a atenuação dolorosa ${ }^{(23)}$.

Administração de sacarose/glucose via oral, é um método empregado minutos antes do procedimento e que auxilia na diminuição do tempo de choro, abrandamento da mímica facial, além de diminuir padrões fisiológicos de sinais vitais, como a frequência cardíaca, por liberar endorfinas que bloqueiam o caminho da dor ${ }^{(8)}$.

A sucção não nutritiva é um reflexo inerente ao recém-nascido, que promove analgesia, conforto e tranquilidade, devido ao aumento da oxigenação, diminuição da frequência cardíaca e do gasto de energia. Pode ser utilizada com o uso do dedo enluvado ou a própria mão do recém-nascido, e parte do mecanismo de diminuição da hiperatividade, duração e intensidade do processo álgico, ajudando desta forma em sua reorganização. Pode ser empregada de maneira isolada ou em associação com outro método ${ }^{(8,24)}$.

A Técnica de distração é um método eficaz e recomendado como uma técnica não farmacológica para alívio da dor, podendo desta forma ser aplicada durante a prática clínica. Um estudo traz que a percepção da intensidade dolorosa durante o procedimento invasivo agudo diminuiu com a aplicação deste método, mostrando-se desta forma eficaz ${ }^{(25)}$.

As limitações encontradas foi a escassez de estudos científicos atualizados sobre a avaliação e manejo da dor em pediatria, diante disso pode-se inferir que o conhecimento científico produzido ainda não está sendo utilizado de forma efetiva dentro da prática clínica, apesar de progressos já terem sido alcançados neste campo. Outra limitação foi a não validação do fluxograma por meio de experts ou prática clínica, no qual será realizado em estudo futuro.

No que tange à contribuição aos profissionais de enfermagem, este estudo procura favorecer a sistematização e a avaliação do evento doloroso, garantir desta forma sua identificação, avaliação e o tratamento do mesmo. Mesmo aqueles profissionais com vasta experiência demonstram não intervir de maneira adequada, mostrando, desta forma, a validade deste instrumento para o dia a dia da prática clínica.

\section{CONCLUSÃO}

Percebe-se que o objetivo deste estudo foi alcançado ao elaborar fluxograma assistencial que possibilita a avaliação, mensuração e tratamento não farmacológico para alívio da dor em crianças e adolescentes. Há fatores que impedem com que os profissionais de enfermagem possam realizar uma avaliação adequada do processo doloroso, como a ausência de um instrumento que sistematize o cuidado. 
Torna-se importante a elaboração de um fluxograma assistencial e este estudo reforça a necessidade de que estudos sejam realizadas, de maneira sistematizada e metodologicamente seguras, no intuito de possibilitar que os instrumentos utilizados viabilizem uma quantificação e qualificação acurada do evento doloroso. Os resultados trarão impactos positivos na gestão do cuidado no intuito de organizar todo o processo, de forma que o profissional de enfermagem possa intervir em momento propício, afim de trazer conforto, assistência segura e humanizada aos pacientes internados em unidades pediátricas.

Estudos futuros são necessários para avaliação da aplicação prática e viabilidade do uso do fluxograma assistencial em diferentes realidades.

\section{REFERÊNCIAS}

1. Santos G, Maranhão DG. Cuidado de Enfermagem e manejo da dor em crianças hospitalizadas: pesquisa bibliográfica. Rev. Soc. Bras. Enferm. Ped [Internet]. 2016 Jun [cited 2019 Sep 07]; 16,(4):44-50. Disponível em: https://sobep.org.br/revista/images/stories/pdf-revista/vol16n1/vol_16_n_1-artigo-de-revisao-2.pdf

2. Azevêdo AVS, Lançoni Júnior AC, Crepald MA. Interação equipe de enfermagem, família, e criança hospitalizada: revisão integrativa. Ciênc. saúde colet. [Internet]. 2017 [cited 2019 Sep 07];22(11). Disponível em: https://doi.org/10.1590/1413-812320172211.26362015

3. Martins SW, Dias FS, Enumo SRF, Paula KMP. Pain assessment and control by nurses of a neonatal intensive care unit. Rev. dor [Internet]. 2013 Mar [cited 2019 Sep 07];14(1):21-6. Available from: http://dx.doi.org/10.1590/S1806-00132013000100006.

4. Candido LK, Tacla MTGMT. Assessment and characterization of pain in children: the use of quality indicators. Rev enferm UERJ [Internet].2015 jul/ago; [cited 2019 Sep 07];23(4):526-32. Disponível em: http://dx.doi.org/10.12957/reuerj.2015.10514

5. Castro CC, Bastos BR, Pereira AKS. Implementação da avaliação da dor como o quinto sinal vital. Rev enferm UFPE on line., Recife. [Internet] 2018 [cited 2019 Sep 07];12(11):3009-14. Disponível em: https://doi.org/10.5205/1981-8963-v12i11a236994p3009-3014-2018.

6. Guedes DMB, Rossato LM, Sposito NPB, Lima DA, Santos B, Meireles E. Avaliação da dor em crianças hospitalizadas. Rev. Soc. Bras. Enferm. Ped. [Internet].2016 Dec [cited 2019 Sep 07].16,(2):68-74. Disponível em: https://sobep.org.br/revista/images/stories/pdfrevista/vol16-n2/vol_16_n_2-artigo_de_pesquisa.pdf

7. Araujo LC, Romero B. Dor: avaliação do $5^{\circ}$ sinal vital. Uma reflexão teórica. Rev. dor [Internet]. 2015 Dec [cited 2019 Sep 07];16(4):291-296. Disponível em: http://dx.doi.org/10.5935/18060013.20150060.

8. Maciel HI, Costa MF, Costa AC, Marcatto JO, Manzo BF, Bueno M. Pharmacological and nonpharmacological measures of pain management and treatment among neonates. Rev Bras Ter Intensiva. [Internet] 2019 [cited 2019 Sep 07];31(1):21-26. Disponível em: 
http://dx.doi.org/10.5935/0103-507X.20190007

9. Silva LM, Silvia AS, Andrade EWOF, Azevedo AP, Costa FWS, Azevedo KPA. A avaliação da dor do recém-nascido realizada pela equipe de enfermagem. Rev. UNINGÁ Review, Maringá. [Internet] 2018 [cited 2019 Sep 07]; 33(1):01-11.

10. Christoffel MM, Castral TC, Daré MF, Montanholi LL, Scochi CGS. Conhecimento dos profissionais de saúde na avaliação e tratamento da dor neonatal. Rev. Bras. Enferm. [Internet]. 2016 June [cited 2019 Sep 07];69(3):552-58. Disponível em: http://dx.doi.org/10.1590/0034-7167.2016690319i.

11. Barros MMA, Luiz BVS, Mathias CV. A dor como quinto sinal vital: práticas e desafios do enfermeiro em uma unidade de terapia. BrJP [Internet]. 2019 Sep [cited 2019 Nov 19];2(3):232-36. Disponível em: http://dx.doi.org/10.5935/2595-0118.20190041.

12. Costa T, Rossato LM, Bueno M, Secco IL, Sposito NPB, Harrison D et al. Conhecimento e práticas de enfermeiros acerca do manejo da dor em recém-nascidos. Rev. esc. enferm. USP [Internet]. 2017 [cited 2019 Sep 07];51:e03210. Disponível em: http://dx.doi.org/10.1590/s1980$220 \times 2016034403210$.

13. Bussotti EA, Guinsburg R, Pedreira MLG. Adaptação cultural para o português do Brasil da escala de avaliação de dor Face, Legs, Activity, Cry, Consolability revised(FLACCr). Rev. Latino-Am. Enfermagem [Internet]. 2015 Aug [cited 2019 Sep 07];23(4):651-659. Disponível em: http://dx.doi.org/10.1590/0104-1169.0001.2600.

14. Oliveira AM, Batalha LMC, Fernandes AM, Gonçalves JC, Viegas RG. Uma análise funcional da Wong-Baker Faces Pain Rating Scale: linearidade, discriminabilidade e amplitude. Rev. Enf. Ref. [Internet]. $2014 \mathrm{Dez}$ [citado 2019 Set 07]; serIV (3): 121-130. Disponível em: http://dx.doi.org/10.12707/RIV14018.

15. Martinez JE, Grassi DC, Marques LG. Análise da aplicabilidade de três instrumentos de avaliação de dor em distintas unidades de atendimento: ambulatório, enfermaria e urgência. Rev. Bras. Reumatol. [Internet]. 2011, Aug [cited 2019 Sep 07];51(4):304-38. Disponível em: http://dx.doi.org/10.1590/S0482-50042011000400002.

16. Brasil. Ministério da Saúde. Protocolos clínicos e diretrizes terapêuticas. Volume 2.Brasília 2010. Disponível em: http://bvsms.saude.gov.br/bvs/publicacoes/protocolos_clinicos_diretrizes_terapeuticas_v2.pdf

17. Distrito Federal. Decreto $\mathrm{n}^{\mathrm{o}}$ 39.546, de 19 de dezembro de 2018. Aprova o Regimento Interno da Secretaria de Estado de Saúde do Distrito Federal. 2018. Disponível em: http://www.sinj.df.gov.br/sinj/Norma/c7d8594440ea48969cee564fafa77865/Decreto_39546_19_12_20 18.html

18. Batalha Luís MC, Sousa AFD. Autoavaliação da intensidade da dor: correlação entre crianças, pais e enfermeiros. Rev. Enf. Ref. [Internet]. 2018 Jun [citado 2019 Set 07];serIV(17):15-22. Disponível em: http://dx.doi.org/10.12707/RIV18002.

19. Valbuena SM, Fernández JA, Fernández IF. Eficacia de una intervención enfermera con realidad virtual en urgencias pediátricas: un ensayo clínico aleatorizado. Revista Enfermería CyL. [Internet]. 2019 [cited 2019 Sep 07] 11-(1):84-94. Disponível em: http://www.revistaenfermeriacyl.com/index.php/revistaenfermeriacyl/article/view/244/213 
20. Bispo CF. Modificações fisiológicas através do riso na interação fisioterapeutapaciente. Fisioterapia Brasil. [Internet] 2018 [cited 2019 Sep 07];[S.1.]6(6):457-461. Disponível em: http://dx.doi.org/10.33233/fb.v6i6.2040.

21. Nascimento JCC. Avaliação da dor em paciente com câncer em cuidados paliativos a luz da literatura. Saúde \& Ciência em Ação - Revista Acadêmica do Instituto de Ciências da Saúde. Ref. [Internet]. $2017 \mathrm{Jan} / \mathrm{Jul} \quad$ [cited 2019 Sep 07]; 3(1). Disponível em: http://revistas.unifan.edu.br/index.php/RevistaICS/article/view/329/248

22. Cruz CT, Gomes JS, Kirchner RM, Stumm EMF. Avaliação da dor de recém-nascidos durante procedimentos invasivos em terapia intensiva. Revista Dor. [Internet] 2016 [cited 2019 Sep 07].;17(3):197-200. Disponível em: https://dx.doi.org/10.5935/1806-0013.20160070

23. Calasans MTA, Maia JMA, Silva JF. A Amamentação como método não farmacológico para o alívio da dor. Revista Enfermagem Contemporânea. Ref. [Internet] 2016 Jul/Dez; [cited 2019 Sep 07];5(2):261-270. Disponível em: http://dx.doi.org/10.17267/2317-3378rec.v5i2.980.

24. Maciel HIA, Costa MF, Costa ACL, Marcatto JO, Manzo BF, Bueno M. Medidas farmacológicas e não farmacológicas de controle e tratamento da dor em recém-nascidos. Rev. bras. ter. intensiva [Internet]. 2019 Mar [cited 2019 Sep 07]; 31(1): 21-26. Disponível em: http://dx.doi.org/10.5935/0103-507x.20190007

25. Costa IB, Dantas DV, Dantas RAN, Oliveira SP, Aiquoc KM, Sarmento SDG. Terapias nãofarmacológicas para o manejo da dor em vítima de queimaduras: uma revisão sistemática. Revista Inspirar movimento \& saúde. [Internet], Abr/mai/jun | 2019, [Cited 2019 Nov 19] 19(2). Disponível em: https://www.inspirar.com.br/wp-content/uploads/2019/07/af_645.pdf 